\title{
Radio, Sub-mm, and X-Ray Studies of Gamma-Ray Burst Host Galaxies
}

\author{
E. Berger \\ Palomar Observatory, California Institute of Technology 105-24, Pasadena, CA 91125
}

\begin{abstract}
The study of gamma-ray burst (GRB) host galaxies in the radio, sub-mm, and X-ray wavelength regimes began only recently, in contrast to optical studies. This is mainly due to the long timescale on which the radio afterglow emission decays, and to the intrinsic faintness of radio emission from star-forming galaxies at $z \sim 1$, as well as source confusion in sub-mm observations; X-ray observations of GRB hosts have simply not been attempted yet. Despite these difficulties, we have recently made the first detections of radio and sub-mm emission from the host galaxies of GRB 980703 and GRB 010222, respectively, using the VLA and the SCUBA instrument on JCMT. In both cases we find that the inferred star formation rates $\left(\sim 500 \mathrm{M}_{\odot}\right)$ and bolometric luminosities (few $\times 10^{12} \mathrm{~L}_{\odot}$ ) indicate that these galaxies are possibly analogous to the local population of UltraLuminous Infrared Galaxies (ULIRGs) undergoing a starburst. However, there is a modest probability that the observed emission is due to AGN activity rather than star formation, thus requiring observations with Chandra or XMM. The sample of GRB hosts offers a number of unique advantages to the broader question of the evolution of galaxies and star formation from high redshift to the present time since: (i) GRBs trace massive stars, (ii) are detectable to high redshifts, and (iii) have immense dust penetrating power. Therefore, radio/sub-mm/Xray observations of GRB hosts can potentially provide crucial information both on the nature of the GRB host galaxies, and on the history of star formation.
\end{abstract}

\section{BACKGROUND: THE STAR FORMATION HISTORY OF THE UNIVERSE}

The formation and evolution of galaxies from high redshift to the current time is a major focus of modern cosmology. This involves mapping the conversion of gas into stars, and the associated buildup of heavy elements. The former is succinctly parameterized by using the light from massive stars as a surrogate for the star-formation rate (SFR; see [1]) and the latter has focused on studies of the intergalactic medium (e.g. [2]) and damped-Ly $\alpha$ systems (e.g. [3]). GRBs can contribute to both these important areas through multi-wavelength studies of their host galaxies, and through absorption spectroscopy of their optical afterglows. Here I address the first issue.

Adelberger \& Steidel (2000)[4] provide a balanced review of the various diagnostics to measure the evolution of SFR: optical (rest-frame UV), far-infrared (FIR), sub-millimeter, and decimeter radio measurements. Each of these techniques has its distinct strengths as well as weaknesses. For example, radio measurements offer superb astrometry but the current VLA sensitivity is only able to identify the tip of the star-formation iceberg [5].
Optical surveys offer the highest sensitivity but are vulnerable to dust extinction and may well miss galaxies forming stars at the most prodigious rate. The FIR and sub-mm approach has maximal sensitivity to dusty galaxies, but it lacks astrometric precision thereby creating a non-trivial bottleneck of requiring detection at other wavelengths; in particular detection by the VLA.

The principal question is the following: Given that each of these techniques provides a restricted view of the cosmic SFR history, and that the optical/UV technique has provided by far the largest sample, can we conclude that optical/UV studies have more or less accounted for the bulk of the cosmic star formation?

Adelberger \& Steidel (2000) seem to think so; a number of other authors, especially those using long wavelength techniques (e.g. $[6,7])$ are of the opinion that this issue is not settled. In the near term, we have reached a stalemate since sub-mm surveys have reached the confusion limit. SIRTF, through its IRAC survey, and ALMA, with its unprecedented $\mu \mathrm{Jy}$ sensitivity will certainly contribute to this critical sub-field of modern astronomy. Nonetheless, since the bulk of the stellar energy is effectively radiated in the FIR band, all these techniques require significant extrapolation to measure the true power radiated by galaxies. In the distant future, one can envis- 


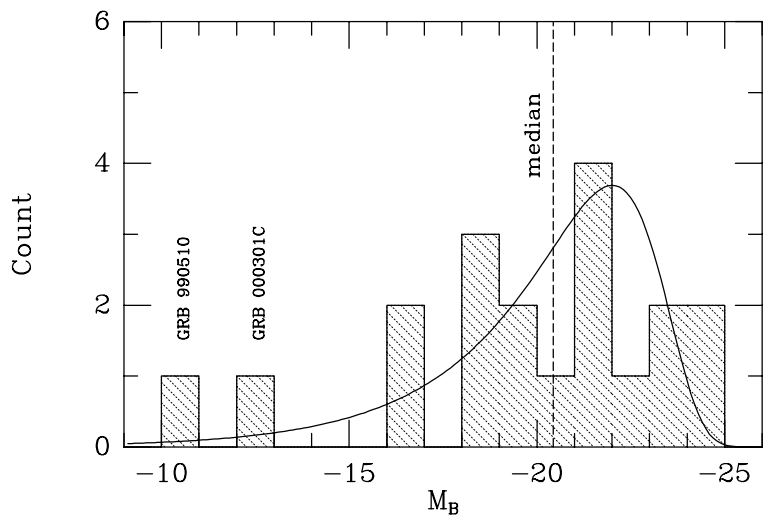

FIGURE 1. Histogram of estimated absolute B-band magnitudes of GRB host galaxies with known redshifts. These restframe magnitudes were computed from the observed R-band magnitudes by approximating the galaxy spectra as $f_{\mathrm{v}} \propto \mathrm{v}^{-1}$. The sample median is $M_{\mathrm{B}}=-20.4 \mathrm{mag}$. The solid curve is a heuristic model representing a luminosity-weighted Schechter function with $M_{*}=-23 \mathrm{mag}$ and $\alpha=-1.6$.

age FIR interferometers as providing the most decisive picture of the cosmic evolution of stellar energy density.

\section{GRB HOST GALAXIES: STRENGTHS}

The host galaxies of GRBs offer a unique perspective into the SFR history of the Universe for the following reasons:

1. The existing data show excellent circumstantial evidence linking GRBs to massive stars (e.g. [8]). More to the point, every well-studied GRB so far has been identified with a host galaxy (Fig. 1).

2. GRBs are so bright that they are detectable to redshifts $>20$ (should they exist; [9]). Thanks to the broad-band afterglow spectrum (X-ray through decimeter radio) not only can the host be accurately localized, but the redshift can also be obtained. Usually $z$ is obtained via optical spectroscopy, though with GRB 990705 [10] we now have a redshift from $\mathrm{X}$-ray spectroscopy of the afterglow.

3. The immense dust-penetrating power of GRBs (only limited by Compton thick column densities) results in a sample of galaxies that is independent of the global dust properties.

4. Again thanks to the afterglow, a GRB host galaxy need only be detected via imaging (with $z$ via absorption spectroscopy) and this has resulted in the faintest luminosity distribution of star-forming galaxies with some hosts 12 magnitudes below $L_{*}$ (Fig. 1). In contrast, current state of the art opti$\mathrm{cal} / \mathrm{UV} / \mathrm{NIR}$ surveys reach $\sim L_{*}$.

\section{GRB HOST GALAXIES: POSSIBLE DRAWBACKS}

It is clear that the sample of GRB hosts offers powerful diagnostics in our quest to decipher the SFR history of the Universe. However, it has two limitations. First, we have to assume that the GRB rate is linearly proportional to SFR. The circumstantial evidence for the association of GRBs with massive stars, and hence SFR is good [11, $12,8,13,14]$. Second, the GRB sample is quite small, especially when compared to the optical/UV sample.

However, the first problem is not as severe as one may think at first glance. All techniques used so far - opti$\mathrm{cal} / \mathrm{UV}$, ISOCAM, sub-mm and decimeter - require large extrapolations (and implicitly, constancy of spectral energy densities) to obtain the bolometric power. Converting this uncertain bolometric estimate to SFR requires detailed assumptions of the IMF of stars and the distribution of ISM in these distant galaxies. The severity of the second problem diminishes when one realizes that the number of securely identified sub-mm galaxies is, as of August 2001, only four! (M. Longair, talk at ESO Lighthouse conference).

\section{THE ORIGIN OF RADIO AND SUB-MM EMISSION FROM GALAXIES}

Having argued that GRBs offer a unique perspective into the cosmic star formation, I now provide a short overview of the underlying sources and emission mechanisms of radio and sub-mm emission from galaxies. The radio luminosity from star-forming galaxies is a combination of synchrotron and thermal emission components, both directly related to the formation rate of massive stars via simple relationships [15]. This is simply due to the fact that radio synchrotron emission comes from electrons accelerated in supernova shocks, the end products of massive stars, and thermal emission comes from HII regions and is dominated by the most luminous (i.e. massive) stars. In addition, since the lifetime of massive stars is $\sim 10^{7}$ years, and the lifetime of the synchrotron emitting electrons is $\sim 10^{8}$ years, radio emission traces the instantaneous SFR [15].

Similarly, sub-mm (and FIR) emission traces star formation since it arises from star-light reprocessed by dust. In this case too the massive stellar population dominates the power output in the host, and therefore the amount of reprocessed radiation. Since the emission in the radio and sub-mm regimes is a tracer of the massive stellar population, it is not surprising that there is a simple relation between the radio and sub-mm luminosities of star forming galaxies. It turns out that this relation is sensitively dependent on redshift [16] [17] [18]. 
One complication to the preceding discussion is the possibility of emission from an obscured AGN, which will contribute to both the radio and sub-mm luminosities of the host. Observations with X-ray satellites can provide an estimate of the fraction of emission (if any) that arises from an active nucleus.

\section{RECENT DETECTIONS: GRB 980703 AND GRB 010222}

Recently, we have detected the host galaxy of GRB 980703 in the radio [12], and the host of GRB 010222 in the sub-mm [13]. Fig. 2 shows the lightcurve of the $8.46 \mathrm{GHz}$ emission from GRB 980703; the flattening at $t>350$ days can only be explained in terms of host emission. We detect similar levels of emission at 1.43 and $4.86 \mathrm{GHz}$. At a redshift of $z=0.966$ these flux levels translate to an emitted luminosity at $1.43 \mathrm{GHz}$ of $L_{\mathrm{em}}(1.43) \approx 4.7 \times 10^{30} \mathrm{erg} \mathrm{sec}^{-1} \mathrm{~Hz}^{-1}$.

What can we learn about the host galaxy of GRB 980703 from the observed radio luminosity? First, the emitted $1.43 \mathrm{GHz}$ luminosity immediately translates to a formation rate of stars more massive than $5 \mathrm{M}_{\odot}, \operatorname{SFR}\left(\mathrm{M}>5 \mathrm{M}_{\odot}\right) \approx 90 \mathrm{M}_{\odot} / \mathrm{yr}$, and a total star formation rate (using the Salpeter IMF) of $\approx 500 \mathrm{M}_{\odot} / \mathrm{yr}$. Second, based on this luminosity and the radio/sub-mm relation, we find that this galaxy is an Ultra-Luminous Infra-Red Galaxy (ULIRG; see [19]), with $L_{\text {FIR }} \approx 10^{12}$ $\mathrm{L}_{\odot}$. A comparison to the properties of radio-selected galaxies at $z \sim 1$ from a survey of the HDF [20], shows that the host of GRB 980703 is by no means an unusual galaxy. On the other hand, a comparison to the opticallyderived SFR ( 20 M $\odot /$ yr; [21]) shows that most of the star formation in this galaxy is obscured.

An alternative explanation for the radio emission is that it originates from an AGN. Surveys of the Hubble Deep Field (HDF), its flanking fields, and the Small Selected Area 13 (SSA13) have shown that approximately $20 \%$ of the radio sources are AGN $[22,23,6]$.

We consider the AGN hypothesis unlikely based on optical spectroscopy. Optical spectra of the source obtained by Djorgovski et al. (1998) show no evidence for an unobscured AGN: high-ionization lines such as Mg II $\lambda 2799$, [NeV] $\lambda 3346$, and [NeV] $\lambda 3426$ are absent, and the [OIII] $\lambda 4959$ to $\mathrm{H} \beta$ ratio is approximately 0.4 , much lower than $[\mathrm{OIII}] / \mathrm{H} \beta>1.3$ for AGN [24]. In addition, AGN have redder colors for similar [OII] EW, relative to normal galaxies [25]. Using the spectrum of GRB 980703 we evaluate the color index, $(41-50) \equiv$ $2.5 \log \left[f_{v}(5000) / f_{v}(4100)\right] \approx 0 \pm 0.1$; an AGN with the same [OII] EW would have a value $>0.3$ [25].

However, it is not possible to rule out the existence of an obscured AGN. Future observations with XMM will

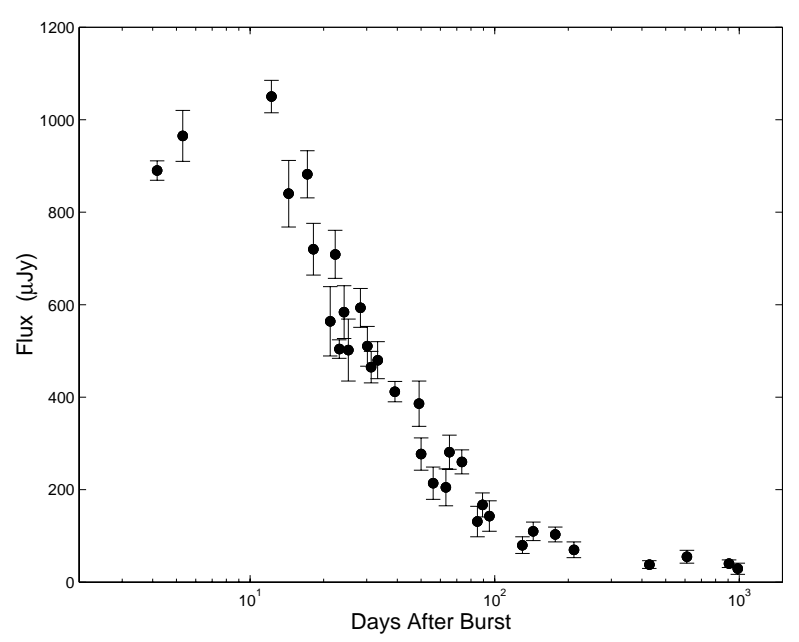

FIGURE 2. Radio light-curve at $8.46 \mathrm{GHz}$, showing the customary initial rise followed by decay of the afterglow of GRB 980703 (Berger et al. 2001). Observations from $\sim 100-$ 300 days after the burst already show signs of flattening, due to the flux contribution from the host, while observations from $t>350$ days directly probe the emission of the host. On these timescales, the afterglow contribution is negligible.

probe this possibility directly.

The high resolution afforded by VLA observations has shown that the GRB-host offset of GRB 980703 is negligible (see Fig. 3), indicating that the burst most probably took place within a nuclear starburst; in this case the resolution of the VLA allows a better offset determination than HST observations [8]. The nuclear starburst origin lends strong support to the collapsar model of GRBs.

The sub-mm detection of the host of GRB 010222 paints a similar picture (Fig. 4). The implied SFR is close to $1000 M_{\odot} / y r$, and the FIR luminosity clearly indicates that this host is also a ULIRG [13].

\section{FUTURE PROSPECTS}

Future radio/sub-mm/FIR studies of GRB hosts will be augmented by X-ray observations in order to assess the importance of obscured AGN in these host galaxies. There is some indication from studies of local ULIRGs [19] that high SFR is usually accompanied by some AGN activity. Thus, the advent of observatories such as the EVLA, SKA, SIRTF, and ALMA, in addition to XMM and Chandra will greatly increase our ability to study the properties of these hosts with greater sensitivity and resolution. For example, with a factor ten increase in resolution and a factor five increase in sensitivity over the current VLA, we will be able to probe scales of approximately 5 mas with the EVLA; for a galaxy at $z \sim 1$ this translates to a physical scale of $150 \mathrm{pc}$. In 


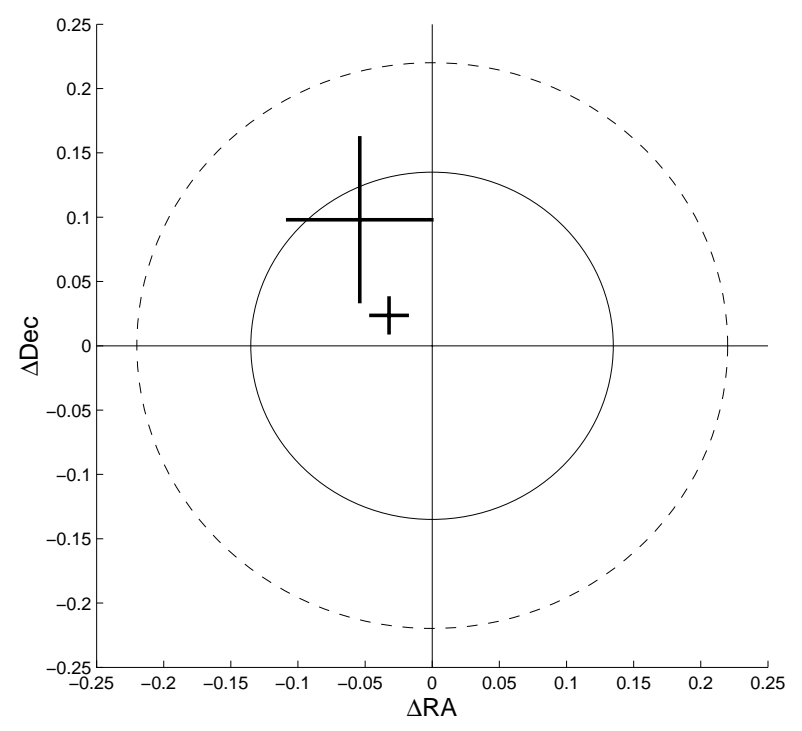

FIGURE 3. The weighted average GRB-host offset in RA and Dec from all VLA observations of GRB 980703 (small cross). The larger cross is the offset measurement from [8]. The solid circle designates the projected maximum source size from the radio observations, and the dashed circle is the optical size from [26]. Clearly the formation of massive stars is concentrated in the central region of the host, and the small offset of the burst from the host center indicates that GRB 980703 occurred in the region of maximum star formation [12]. This points to a link between GRBs and massive stars.

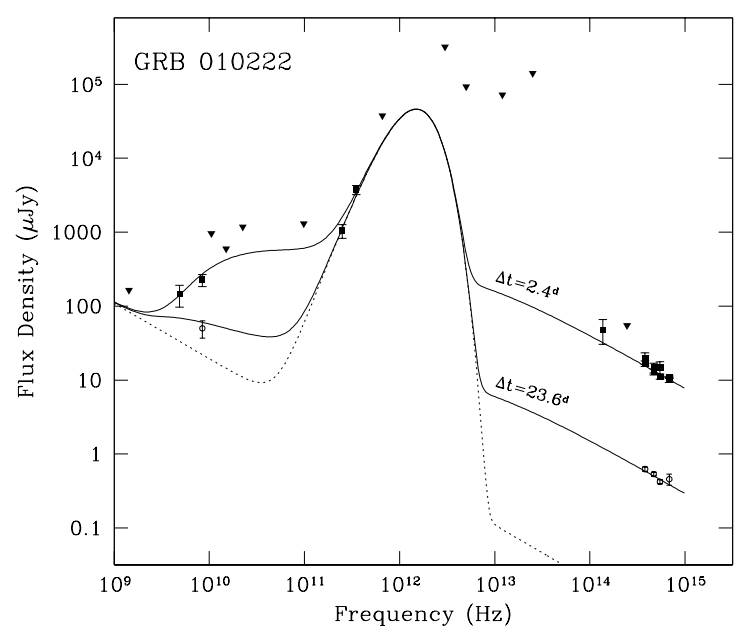

FIGURE 4. Spectral energy distribution of the host+afterglow emission from GRB 010222. The dashed line is the contribution of the host, and the solid line is the fading afterglow. The sub-mm flux densities at 250 and 350 $\mathrm{GHz}$ point to $\mathrm{SFR} \sim 750 \mathrm{M}_{\odot} / \mathrm{yr}$, and a ULIRG host galaxy. From [13] addition, EVLA will detect galaxies with a total SFR as low as $50 \mathrm{M}_{\odot} / \mathrm{yr}$ at $z \sim 1$.

The potential of a GRB-selected galaxy sample is immense and unique. The dust-penetrating power of GRBs and their broad-band afterglow emission, offer a number of unique diagnostics: the obscured star formation fraction, the ISM within the disk, the local environment of the burst, and global and line-of-sight extinction, to name a few. In addition, GRBs allow us to select a wide range of galaxies independent of their emission propertied in any wavelength regime, and in addition they supply redshift information for these galaxies; the lack of accurate redshifts is one of the main problems of sub-mm studies of high-redshift galaxies.

It appears, therefore, that the numerous and detailed optical studies of GRB hosts are only the tip of the iceberg in our understanding of galaxies at high redshifts.

\section{REFERENCES}

1. Madau, P., et al. 1996, MNRAS, 283, 1388.

2. Rauch, M. 1998, ARAA, 36, 267.

3. Storrie-Lombardi, L. J. \& Wolfe, A. M. 2000, ApJ, 543, 552.

4. Adelberger, K. L. \& Steidel, C. C. 2000, ApJ, 544, 218.

5. Ramaprakash, A. N., et al. 1998, Nature, 393, 43.

6. Barger, A. J., Cowie, L. L., \& Richards, E. A. 2000, AJ, 119, 2092.

7. Peacock, J. A., et al. 2000, MNRAS, 318, 535.

8. Bloom, J. S., Kulkarni, S. R., \& Djorgovski, S. G. 2001, submitted to AJ; astro-ph/0010176.

9. Lamb, D. Q. \& Reichart, D. E. 2000, ApJ, 536, 1.

10. Amati, L., et al. 2000, Science, 290, 953.

11. Piro, L., et al. 2000, Science, 290, 955.

12. Berger, E., Kulkarni, S. R., \& Frail, D. A. 2001, ApJ, 560, 652.

13. Frail, D. A., et al. 2001, Accepted to ApJ; astro$\mathrm{ph} / 0108436$.

14. Reichart, D. E. 2001, Submitted to ApJL; astro$\mathrm{ph} / 0107546$.

15. Condon, J. J. 1992, ARAA, 30, 575.

16. Carilli, C. L. \& Yun, M. S. 1999, ApJ, 513, L13.

17. Carilli, C. L. \& Yun, M. S. 2000, ApJ, 530, 618.

18. Dunne, L., Clements, D. L., \& Eales, S. A. 2000, MNRAS, 319, 813.

19. Sanders, D. B. \& Mirabel, I. F. 1996, ARAA, 34, 749+.

20. Haarsma, D. B., et al. 2000, ApJ, 544, 641.

21. Djorgovski, S. G., et al. 1998, ApJ, 508, L17.

22. Richards, E. A., et al. 1999, ApJ, 526, L73.

23. Richards, E. A. 2000, PASP, 112, 1001.

24. Rola, C. S., Terlevich, E., \& Terlevich, R. J. 1997, MNRAS, 289, 419.

25. Kennicutt, R. C. 1992, ApJ, 388, 310.

26. Holland, S., et al. 2001, submitted to A\&A. astro$\mathrm{ph} / 0103058$. 